\title{
Design of the Lightweight Structure Inspired by Beetle Elytra and Its Mechanical and Thermal Properties
}

\author{
C. GUO ${ }^{1, a, *}$, T. XU $U^{1, b}, Y . Z H O U^{1, c}$, D. LI ${ }^{1, d}$ \\ ${ }^{1}$ Institute of Bio-inspired Structure and Surface Engineering, Nanjing University of Aeronautics and \\ Astronautics, Nanjing 210016, China \\ aguozc@nuaa.edu.cn, b425151303@qq.com, 'chhouyizhouyi12@yahoo.com.cn, \\ lidongqau@126.com \\ ${ }^{*}$ Corresponding author
}

Keywords: Elytra, Microstructure, Bio-Inspired Structure, FEM, Epoxy Resin Based Carbon Fibre Composite.

\begin{abstract}
Based on the microstructure of the cross section of the beetle elytra, a kind of bio-inspired lightweight structure with fibre-winding pattern was designed and made by the carbon fibre material. The mechanical and thermal properties of the lightweight structure were studied with finite element method. At the same time, the quasi-static compression experiment was carried out. The experimental result and the finite element analysis result were compared and analysed, which proved the effectiveness of the finite element analysis. Additionally, the mechanical properties of two kinds of structure units with the different fibre winding pattern were analysed and compared, the result showed the structure with two layers of different fibre-winding patterns was better to improve its mechanical performance. Also, thermal characteristics of the structure showed its thermal protection property was favorable.
\end{abstract}

\section{Introduction}

As technology advances, many industries put forward the new requirements to the materials (e.g., higher specific intensity, specific rigidity, damage resistance), and many traditional materials and structures can not meet industry requirements again. However, natural biological materials formed many unique features through 35 million years of evolution in order to adapt to the environment and satisfy the functional requirements with capabilities such as strong toughness, functional adaptability and wound healing capabilities, et al, which are unsurpassed by conventional artificial materials [1]. Now people try to get inspiration from nature to develop new bio-inspired composite materials and structures. Since the 1980s, significant progress has been made in understanding the structures and its special functions of the biological materials and the bionic research. Mehmet et al. [2] studied the composite structure and the mechanical mechanism of pearl shell layer's cross-section and developed the new ceramic matrix polymer and ceramic-metal composites, whose fracture toughness improved $40 \%$ more than the conventional ones. According to the bamboo structure feature with dense outside and sparse inside, Sun et al. [3] manufactured a novel kind of double-layer carbon fibre materials whose bending strength and electrical properties were significantly improved comparing with the traditional materials. Gordon et al. [4] used the composite columns, plates and sandwich materials to imitate the spiral structure found in wood cells and made glass fibre/epoxy composite materials with the significantly improved fracture toughness.

Beetle is a very large population in the nature, and their former wing in the evolutionary process slowly evolved into elytra with high strength and fracture toughness [5-6]. Now, the beetle has become a good bionic object for geometric design and material optimum of the novel materials and structures. Chen et al. [7-9] studied the cross section structure and surface microstructure of hercules beetle, and pointed out that the elytra is a sandwich structure with small columns as bridge peers to connect the top and bottom layers. Chen also [10] fabricated a kind of composite laminate with biomimetic fibre helicoidally rounding holes and examined the extrusion strength of the hole. Yang [11,12] observed the cross-section microstructure of cybister elytra and measured the tensile 
strength of the elytra, the experimental results showed that the elytra cross section is composed of cavities, fibre bundles that connect inner layers to the outer cuticular layers, several chitin fibre layers and a dense black epicuticle. The tensile test of the elytra showed that it has high specific strength.

In this paper, on the basis of the microstructure of the cross section of Cybister (Cybister tripunctatus Olivier) elytra, a bio-inspired structure with the fibre winding pattern was designed, its mechanical and thermal properties were studied with FEM, and samples made of the epoxy resin based carbon fibre composite were built, the quasi-static compression experiment was carried out to verify the FEM result. Additionally, the effects of the different fibre winding patterns on structure mechanical properties were analysed, the results showed the structure with two different fibre-winding patterns has better mechanical performance than that of the structure with only one fibre-winding pattern. On the other hand, thermal characteristics of the structure showed its thermal protection property is favorable.

\section{Bio-inspired Design of the Lightweight Structure}

\section{Microstructure Features of the Cross Section of Cybister Elytra}

The microstructure of the cross section and the mechanical properties of the beetle elytra have been studied by many researchers [13-18]. Fig.1 is the microstructure of the cross section of cybister elytra, it is a typical sandwich structure, and composed of cavities, fibre bundles, chitin fibre layers and a dense black epicuticle. The fibre bundles are braided in the parallel, perpendicular and spatial helix ways, which improve the mechanical properties of elytra, such as intensity, toughness, and peeling resistance, et al. According to references [11,12], the thickness of the epicuticle, a single chitin fibre layer and the average diameter of cavities are 12, 2, 80-95 $\mathrm{m}$, respectively, the cavities in the elytra effectively reduce the structure weight over a span of about $250 \mu \mathrm{m}$. The Cybister elytra have a duty ratio (cavity area to elytra area) of about $22 \%$ and density of $0.89 \times 10^{3} \mathrm{~kg} / \mathrm{m}^{3}$, while their average hardness and Young's modulus are 0.48 and $8.21 \mathrm{GPa}$ respectively, and their transverse and longitudinal tensile strengths are as high as $169.2 \pm 22.5$ and $194.5 \pm 23.4 \mathrm{MPa}$ respectively.

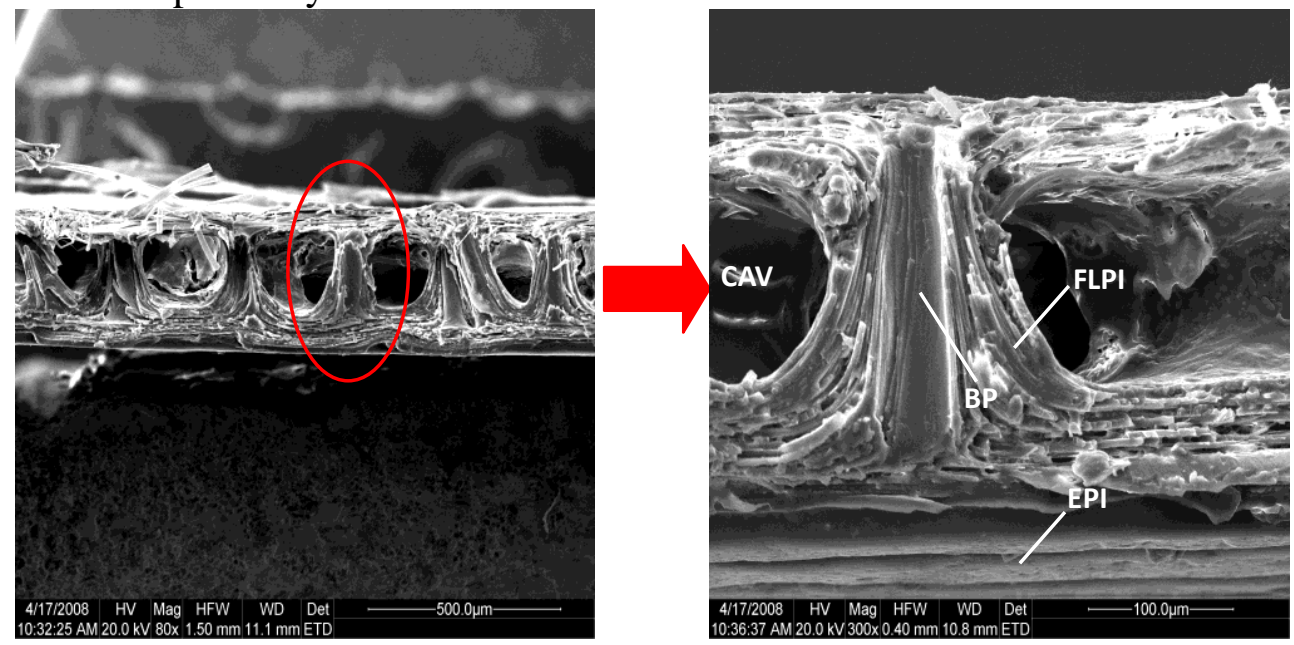

Fig.1 Microstructures of the cross section of elytra. EPI, Epicuticle; FL, Fibre layers; CAV, Cavity; $\mathrm{BP}$, Bridge pier

\section{Structural Design Inspired by Beetle Elytra}

According to the morphological features of the cybister elytra, also referring to the model suggested by Chen [8], a bio-inspired lightweight structure featured by cavities and hollow columns which are composed of fibres braided in different patterns was suggested, and the number of fibre layers and the orientations of fibres were shown in the cross-section of column (Fig. 2). 


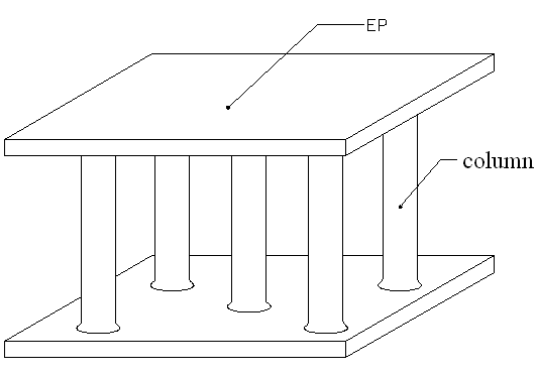

(a)

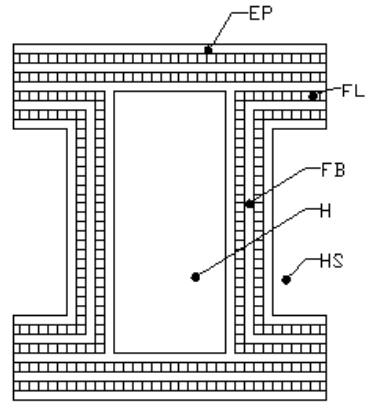

(b)

Fig.2 Elytra inspired lightweight structure. (a) The bio-inspired structure; (b) The cross-section structure of single hollow column. EP: Laminated plate; FL: spiral fibre layer; FB: vertical fibre layer; HS: cavity; H: column holes

\section{The Mechanical Properties of the Bio-inspired Lightweight Structure}

\section{Quasi-static Compression Analysis}

\section{FEM analysis of compression strength of the structure.}

(1)Finite Element Modeling

To simplify FEM model, firstly, the structure composed of layers of fibres with the orientation paralleled to the axis of column and paralleled horizontally in the upper and bottom planes was built and analysed. The overall size of the model is $30 \times 30 \times 20 \mathrm{~mm}^{3}$, and the thickness of the upper and bottom plane, the inner diameter of the small column and the wall thickness are $1.5 \mathrm{~mm}, 3.5 \mathrm{~mm}$ and $0.5 \mathrm{~mm}$, respectively. 3D model of the structures was built with pro/E software and then transferred into ANSYS Workbench environment, the finite element type was Solid 186, and the total numbers of nodes and elements of the structure were 60659, 31215 respectively, as shown in Fig. 3. The material of the model was epoxy resin based carbon fibre composite, the same material was used to build the structure samples. The material constants were set as Table 1.

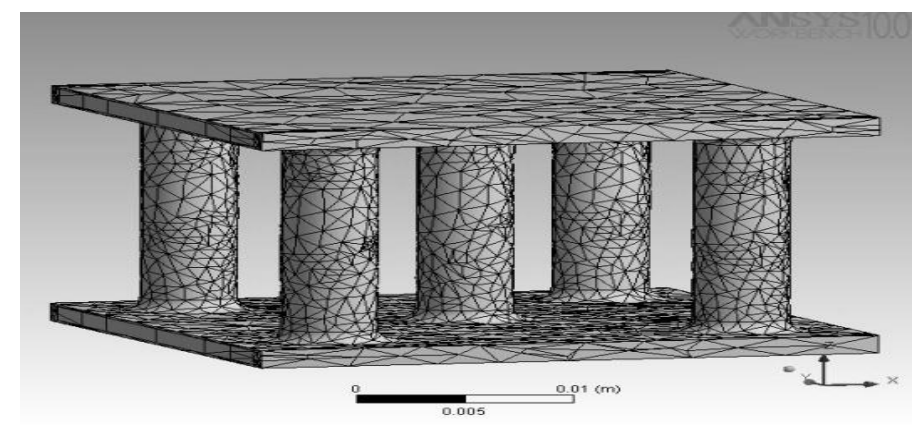

Fig.3 The finite element model of the bio-inspired structure

Tab.1 The material properties of the model

\begin{tabular}{ccccc}
\hline material & $\begin{array}{l}\text { Elastic } \\
{[\mathrm{GPa}]}\end{array}$ & modulus & $\begin{array}{l}\text { Density } \\
{\left[\mathrm{g} / \mathrm{cm}^{3}\right]}\end{array}$ & $\begin{array}{l}\text { Tensile } \\
{[\mathrm{MPa}]}\end{array}$ \\
\hline carbon fibre & $253^{*}$ & 1.78 & $4980^{*}$ \\
\hline SU810 epoxy resin & 2.5 & 1.2 & 55 \\
\hline
\end{tabular}

* Elastic modulus and Tensile strength along the length of carbon fibre

(2)Load and boundary constraints

Similar to the load and boundary constraints in the quasi-static compression experiment of the honeycomb structure [19], displacement was applied as a load to the top surface of the model step by step. Here, the number of load steps should be chosen reasonably. The model bottom was fixed for all six degrees of freedom (DOFs). 


\section{(3)Results Analysis}

The compression force corresponding to the displacement of each sub-step was obtained through finite element analysis, then, the force-displacement curve was determined (Fig. 4). It can be seen that the compression force initially increased with the displacement, however, when the compression force reached the critical yield limit, the force changed little as the displacement continued to increase, demonstrating the structure has already reached the elastic-plastic stage, and its compression limit load was $12.7 \mathrm{KN}$. According to the equation $\sigma=\mathrm{F} / \mathrm{A}$, where $\mathrm{F}$ is the compression limit load $(\mathrm{N})$, and $\mathrm{A}$ is the area to bear the load $\left(\mathrm{mm}^{2}\right)$, the compression strength of the structure was 14.1 $\mathrm{MPa}$. Taking the average density of the structure into account, the specific compression strength was $40.3 \mathrm{kN} \mathrm{m} / \mathrm{kg}$.

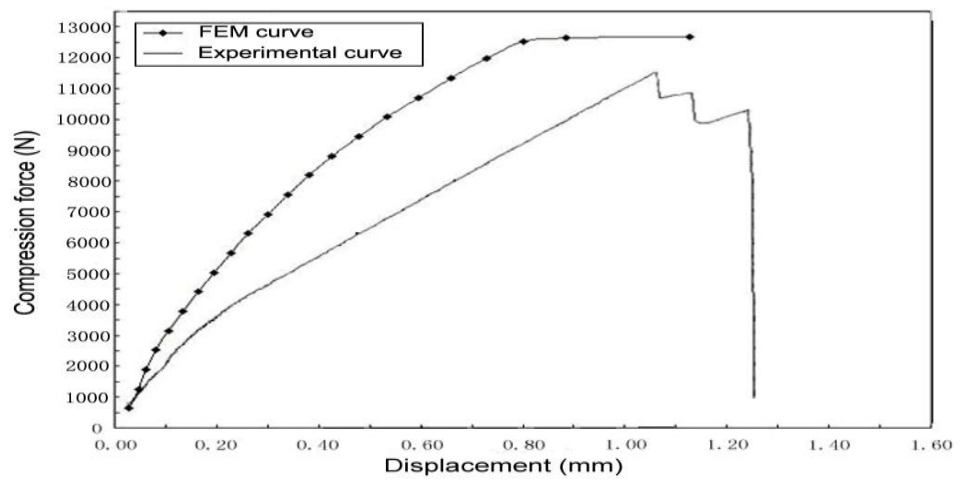

Fig.4 Comparison of the experimental results with the finite element analysis

Experimental Verification of FEM Analysis. In order to verify the reliability of the finite element analysis, the structural samples were built from same material (Fig. 5), and a quasi-static compression experiment was performed on an electronic universal testing machine with load changing between 0 and $100 \mathrm{KN}$ (Changchun, China).

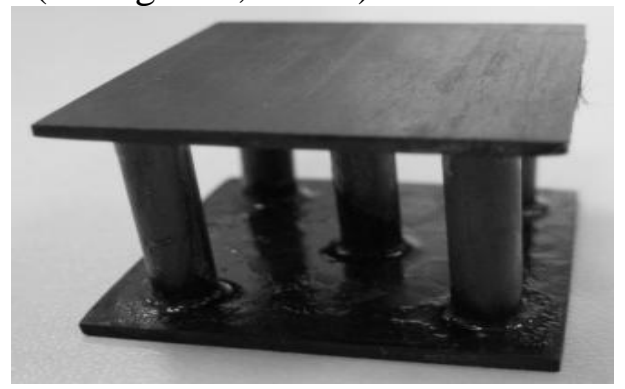

Fig.5 Structure sample

The quasi-static compression was a single-direction force produced by moving the pressure head with a constant velocity of $1 \mathrm{~mm} / \mathrm{min}$. The force produced on the pressure head and the displacement due to the compression deformation of the sample were recorded and monitored by sensors. The test results showed that the compression limit load was $11.6 \mathrm{KN}$. Comparing with the FEM result, the error was $9.5 \%$, the difference mainly came from the glue connections between the columns and the top/bottom plates in the structural samples, which was not considered in the FEM model, but definitely reduced the compression ability of samples. Fig. 4 compared the experimental curve with the FEM analysis curve. Although there was a difference between the experimental and analytical results, especially the force of the experimental curve sharply declined after reaching a maximum owing to the structural fracture, while the force indicated by the analytical curve declined gently, it was seen that the experimental results were in accordance with the analytical results at the first stage, demonstrating the effectiveness and reliability of the finite element method analysis.

\section{Shear Strength Analysis}

FEM Model. The same 3D model in section 2.1 was used again. According to the reference [20], 
the bottom plane of the model was fixed for all six degrees of freedom, and the upper plane was defined as the rigid plane without any deformation, and in-plane horizontal force was applied as the shear force in Fig. 6(a).The material constants were just the same as in Table 1.

Results Analysis. The force and the corresponding displacement in each sub-step were obtained through finite element analysis, and the shear force-displacement curve was determined as shown in Fig. 6(b). According to the equation $\tau=F_{\max } / A$ and equation $G_{e q}=1000 \times \delta \times \theta / A$, where $F_{\max }$ is the maximum shear force $(\mathrm{N})$, and $\mathrm{A}$ is the original area to bear the force $\left(\mathrm{mm}^{2}\right), \delta$ is the original thickness of the sample ( $\mathrm{mm})$, and $\theta$ is the linear slope of the straight-line fitting of the linear part of the curve $(\mathrm{N} / \mathrm{mm})$, the shear strength of the bionic structure $\tau$ was $1.8 \mathrm{MPa}$, and the equivalent shear modulus $\mathrm{G}_{\text {eq }}$ was $94.1 \mathrm{Mpa}$.

The FEM results showed that the bio-inspired structure had excellent mechanical properties, its specific compression strength was $40.3 \mathrm{kN} \mathrm{m} / \mathrm{kg}$, which was much higher than the values of $4 \sim 16$ $\mathrm{kN} \mathrm{m} / \mathrm{kg}$ for stainless-steel hollow-sphere foam and Al foam [21], and its shear strength was 1.8 $\mathrm{MPa}$, shear modulus was $94.1 \mathrm{Mpa}$, both were greater than the values of T722 Nomex honeycomb structure ( $\tau=1.56 \mathrm{Mpa}$ and $\left.\mathrm{G}_{\text {eq }}=59.2 \mathrm{Mpa}\right)$ [22].

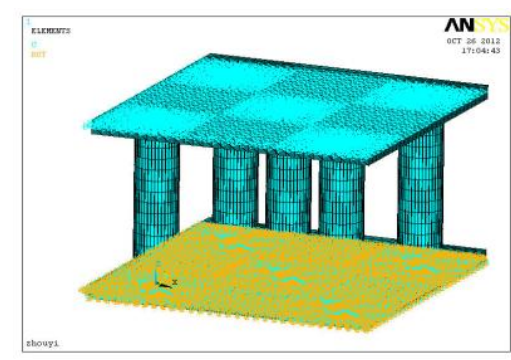

(a)

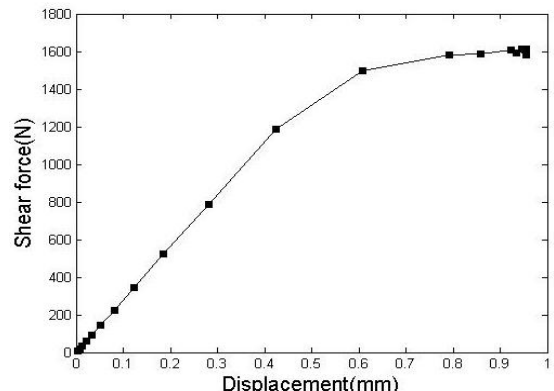

(b)

(a) FEM of the structure; (b) The shear force-displacement curve of the structure

Fig.6 FEM analysis of the shear strength of the bio-inspired structure

\section{The Effect of Different Fibre Winding Patterns on the Mechanical Properties of the Structure}

As mentioned above, the fibre bundles of cybister's elytra are braided in the parallel, perpendicular and spatial helix ways, which improve the mechanical properties of elytra, and the designed bio-inspired structure in this paper also was braided in fibres with two different patterns. So, it is necessary to further analyse the effect of the fibre winding patterns on the mechanical properties of the structure.

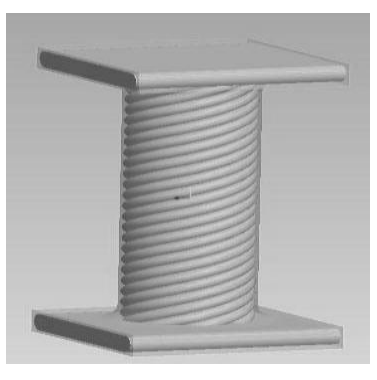

(a)

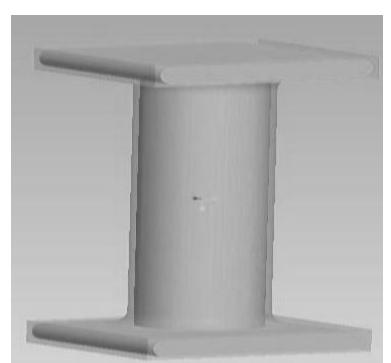

(b)

Fig.7 Different fibre winding patterns: (a) vertically paralleled fibre + spatial helical fibre; (b) two vertically paralleled fibres

3D FEM model. Here, the small hollow column with two different fibre winding patterns was built as the FEM model, and two fibre winding patterns included the parallel and the spatial helical patterns. In Fig.7, the column was wrapped in two layers of fibres, (a) was composed of one layer of vertically paralleled fibre and one layer of the spatial helical fibre, and the helix angle was $65^{\circ}$. (b) was composed of two layers of vertically paralleled fibres. The finite element type was Solid 186, the 
material was the same as that of the bionic structure, material constants can be referred to Table 1 .

Comparison of the Mechanical Properties of Two Columns. The quasi-static compression and shear strength of two columns were analysed and compared with FEM. The load and boundary constraints of the quasi-static compression analysis were the same as those mentioned in section 2.1, and the load and boundary constraints of the shear strength analysis were defined as [23]: the top plate of the model was fixed for all six degrees of freedom, while the bottom plate was fixed axially, and horizontally shear force was applied to the middle part of the model. The compression force-displacement curve and the shear force-displacement curve were determined as shown in Fig. $8 \sim 9$.

It can be seen from Fig.8 Fig.9 that the shear strength of the column (a) was greater than that of the column (b), while the compression strength of the former was smaller than that of the latter, demonstrating the fibre braided in the vertically paralleled ways is more suitable to bear the compression force. Thus, the bio-inspired structure with two different braiding patterns could possess both mechanical advantages, and has better mechanical properties.
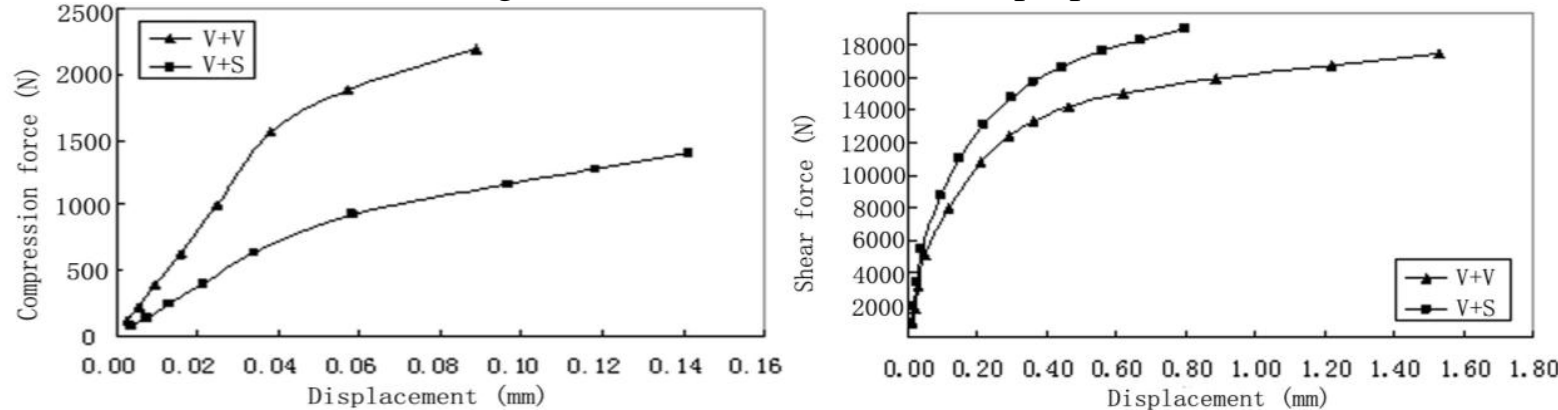

Fig.8 The compression force-displacement curve Fig.9 The shear force-displacement curve

\section{The Thermal Property of the Bio-inspired Lightweight Structure}

Considering the potential application in the field of aerospace, the thermal characteristics of the bio-inspired lightweight structure should be investigated, too.

\section{Thermal Boundary Conditions and Load}

Initial Thermal Condition. The initial temperature of the lightweight structure was defined as the ambient temperature $20^{\circ} \mathrm{C}$ ).

Boundary Conditions. There are three patterns of heat transfer: conduction, convection and emission. Because temperature rise of the structure is low, the dissipating heat due to emission is omitted.

Heat Transfer Coefficient. It was assumed that the structure was put in a closed room, and had no heat exchange between the structure and outside environment. The convection belongs to natural convection, and the heat transfer coefficient is $\left.1 \sim 10 \mathrm{~W} /\left(\mathrm{m}^{\circ}{ }^{\circ} \mathrm{C}\right)\right)[24]$, which is set as $5 \mathrm{~W} /(\mathrm{m} \bullet)$ in the FEM analysis.

Conduction Coefficient. The conduction coefficients for the epoxy resin based carbon fibre composite is $0.82 \mathrm{~W} /\left(\mathrm{m} \cdot{ }^{\circ} \mathrm{C}\right)$ [25].

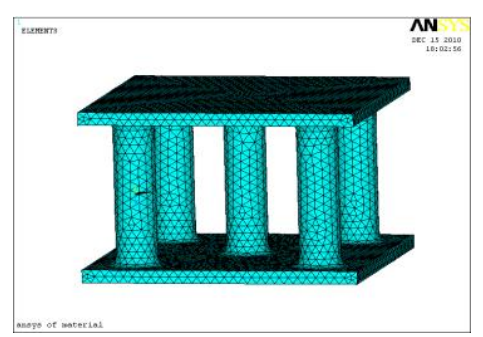

(a) Thermal model of the

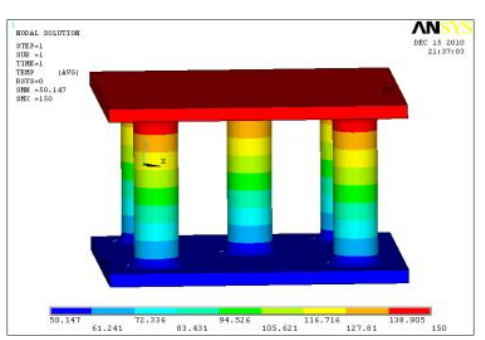

(b) Temperature distribution in the steady state

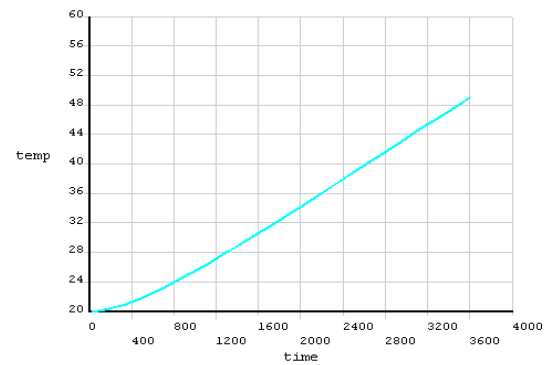

(c) The curve of temperature increase with time

Fig.10 The thermal characteristics of the bio-inspired lightweight structure 
Thermal Load. Considering the carbon fibre material should be used under $200^{\circ} \mathrm{C}$ ) in order to maintain its normal mechanical properties [26], the thermal load applied to the upper surface of the structure was set as $150^{\circ} \mathrm{C}$ ).

\section{Thermal Analysis}

ANSYS13.0 was utilized to analyze its thermal characteristics and calculate its temperature distribution. The finite element model was shown as Fig. 10(a), it contains 22353 SOLID90 elements, and 44180 nodes. The temperature distribution of the structure in the steady state and the temperature increase with respect to time were shown in Fig.10 (b) and Fig. 10(c).

It can be seen that the highest temperature of the bottom surface of the structure is $50.15^{\circ} \mathrm{C}$ ), and the temperature increases to $45.7^{\circ} \mathrm{C}$ ) after $3600 \mathrm{~s}$, not yet reaches the heat balance, demonstrating the thermal protection property of the elytra-inspired structure is favorable.

\section{Conclusions}

A beetle elytra-inspired lightweight structure was designed and made of carbon fibre material, and its mechanical properties and thermal characteristics were studied with finite element method. At the same time, the quasi-static compression experiment was carried out to verify the effectiveness of the finite element analysis. The mechanical investigation of the bio-inspired structure demonstrated that it has excellent mechanical properties, its specific compression strength is $40.3 \mathrm{kN} \mathrm{m} / \mathrm{kg}$, much higher than the values of $4 \sim 16 \mathrm{kN} \mathrm{m} / \mathrm{kg}$ for stainless-steel hollow-sphere foam and $\mathrm{Al}$ foam, and its shear strength is $1.8 \mathrm{MPa}$, shear modulus is $94.1 \mathrm{Mpa}$, both are greater than the values of T722 Nomex honeycomb structure $(\tau=1.56 \mathrm{Mpa}$ and Geq $=59.2 \mathrm{Mpa})$. Additionally, the mechanical properties of two kinds of structure units with the different fibre winding patterns were analysed and compared, the results showed the structure with two layers of different fibre-winding patterns is better to improve its mechanical performance. Also, thermal characteristics of the structure showed its thermal protection property is favorable.

\section{Acknowledgment}

This work was supported by the National Basic Research Program of China (2011CB302106), the National Natural Science Foundation of China (51175249, 51475230), the Ph.D. Programs Foundation of Ministry of Education of China (20123218110010), and the Aero-Science Foundation of China (2013ZF52072).

\section{References}

[1] Z. D. Dai, Y. F. Zhang, X. C. Liang and J.R. Sun, Coupling between elytra of some beetles: Mechanism, forces and effect of surface texture, Sci. China. Ser. C: Life Sci., 2008, 51, p.894-901.

[2] S. Mehmet, K. E. Gunnison and M. Yasrebi, Seashells as a natural model to study laminated composites, Proc. of the American Society for Composites, 5th Technical Conference, Lancaster Pennsylvania, USA, 1990, p.176-183.

[3] S. J. Sun, Z. M. Wang and M. D. Zhang, CF/Cu Composite materials Containing Fe or Ni, Acta Mater. Comp. Sin., 1990, 7, p. 30-34, 39. (in Chinese)

[4] J. E. Gordon and G. Jeronimidis, Composites with high work of fracture, Phil. Trans. R. Soc. Lond., A, 1980, 294, p.545-550.

[5] B. Chen, X. H. Peng and J. H. Fan, Round-hole-fibre distribution in insect cuticle and biomimetic research, JSME Inter.J. C: Mech. Sy., Machine Elements and Manufacturing, 2004, 47, p.1128-1132.

[6] C. Guo, W. W. Song and Z. D. Dai, Structural design inspired by beetle elytra and its 
mechanical properties, Chin. Sci. Bull., 2012, 57, p.941-947.

[7] J. X. Chen, Q. Q. Ni and E. Yasuhisa, Distribution of trabeculae and elytral surface structures of the horned beetle (Allomyrina dichotoma), Entomol. Sin., 2002, 9, p.55-61.

[8] J. X. Chen, Q. Q. Ni, Y. L. Xu and M. Iwamoto, Lightweight composite structures in the forewings of beetles, Compos. Struct., 2007, 79, p.331-337.

[9] J. X. Chen, G. Z. Dai, Y. L. Xu and M. Iwamoto, Basic study of biomimetic composite materials in the forewings of beetles, Mater. Sci. Eng.: A, 2008, 483-484, 625-628.

[10]B. Chen, D. G. Yin, J. H. Fan, et al, Fibre helicoidally rounding hole of tumblebug's elytra and extrusion strength of biomimetic composite, Mater. Res. Innov., 2013, 17, p.17-21.

[11]Z. X. Yang, Z. D. Dai and C. Guo, Morphology and mechanical properties of cybister elytra, Chin. Sci. Bull., 2010, 55, p.771-776.

[12]Z. X. Yang, Biomimetic Research on Microstructures, Mechanical Properties and Coupling Mechanism of Beetle Elytra, PhD thesis, Nanjing university of Aeronautics and Astronautics, Nanjing, China, 2009, p.26-71 (in Chinese).

[13]J. W. L. Beament, Wetting properties of insect cuticle, Nature, 1960,186, p.408-409.

[14]A. R. Parker and C. R. Lawrence, Water capture by a desert beetle, Nature, 2001, 414, 33-34.

[15]S. N. Gorb and P. J. Goodwyn, Wing-locking mechanisms in aquatic heteroptera, J. Morphol., 2003, 257, p.127-146.

[16]J. F. V. Vincent and U. G. K. Wegst, Design and mechanical properties of insect cuticle, Arthropod Struct. Dev., 2004, 33, p.187-199.

[17]Y. B. Cohen, Biomimetics-using nature to inspire human innovation, Bioinspir. Biomim., 2006,1, p.1-12.

[18]B. Bhushan, Biomimetics: lessons from nature-an overview, Phil. Trans. R. Soc. A, 2009, 367, p.1445-1486.

[19]C. F. Choon, B. C. Gin and K. S. Leong, Mechanical properties of Nomex material and Nomex honeycomb structure, Compos. Struct., 2007, 80, p.588-594.

[20]ISO 1922, Rigid cellular plastics-determination of shear strength, 2001 (E)

[21]A. Rabiei and L. J. Vendra, A comparison of composite metal foam's properties and other comparable metal foams, Mater. Lett., 2009, 63, p.533-536.

[22]Y. Q. Luo and W. Hao, Study on Properties of T722 Nomex Honeycomb. Hi-Tech. Fibre. \& Application, 2009, 34, p.34-37.(in Chinese)

[23]X. Han and N. H. Hao, Finite Element Analysis of Steel Tubes Shearing, Machine Building \& Automation, 2009, 38, p.15-17. (in Chinese)

[24] Y. Zhang and E. Z. Guo, Heat Transfer, Southeast University Press, Nanjing, 2004(in Chinese).

[25] N. B. Yang and Zhang Y N, Composite materials for aircraft structures, Aviation Industry Press, Beijing, 45, 2002. (in Chinese).

[26] M. Chen, Y. C. Long, Z. Y. Chen, W. Zhang and Z. G. Yang, Test and Numerical Simulation of Failure Strength for the Composite Material at High Temperature, Mater. Rev., 2010, 24, p.81- 84. (in Chinese) 